\title{
Inequality, Welfare and Monotonicity
}

\author{
by \\ Yoram Amiel \\ Ruppin Institute, Israel \\ and \\ Frank Cowell \\ STICERD, London School of Economics and Political Science
}

The Toyota Centre

Suntory and Toyota International Centres for

Economics and Related Disciplines

Discussion Paper

London School of Economics and Political Science

No.DARP/29

Houghton Street

London WC2A $2 \mathrm{AE}$

February 1997

Tel.: 020-7955 6678

This research was partially supported by an ESRC Grant No.R000235725. We are grateful to Leonardo Gasparini and Peter Lambert for comments. 


\section{Abstract}

We stablish a general relationship between the standard form of the individualistic social-welfare function and the "reduced-form" version that is expressed in terms of inequality and mean income. This shows the relationship between the property of monotonicity and the slope of the equity-efficient trade-off. Particularly simple results are available for a large class of inequality measures that includes the Gini. These results do not require differentiability of the social-welfare function.

Keywords: Inequality, social welfare, monotonicity.

JEL Nos.: C13, D63.

(C) by the authors. All rights reserved. Short sections of text, not to exceed two paragraphs, may be quoted without explicit permission provided that full credit, including $\odot$ notice, is given to the source.

Contact address: Contact address: Professor Frank Cowell, STICERD, London School of Economics and Political Science, Houghton Street, London WC2A 2AE, UK. Email: f.cowell@Ise.ac.uk 


\section{Introduction}

Inequality indices embody explicitly or implicitly social values on income distribution. So it is common for those who are concerned with comparing distributional changes over time or between economies to draw conclusions from comparisons of estimates of average income and inequality. However the relationship between intuitive comparisons in terms of say, mean income and the Gini coefficient, and formal principles of social welfare is not always made clear. The present paper focuses upon this relationship by characterising the shape of the equity-efficiency trade-off..

This trade-off is at the heart of much theoretical and applied research. For example, it is become standard practice to use the obvious two-way relationship between certain classes of inequality indices and their associated social-welfare functions to infer propositions about distributional rankings and the ethical meaning of inequality measures. ${ }^{1}$ In addition there is an interest in constructing socialwelfare functions for inequality measures with principally pragmatic appeal: for example, there have been a number of attempts to formalise a social-welfare function on the basis that the Gini coefficient is the appropriate index of inequality. ${ }^{2}$ The precise method of doing this formalisation does not concern us here, except as illustrations of our approach. We want to examine in a general way what may

\footnotetext{
${ }^{1}$ See for example Atkinson (1970), Blackorby and Donaldson (1978, 1980), Kolm (1976a, 1976b).

${ }^{2}$ See for example Chipman (1974), Katz (1972), Kondor (1975), Lambert (1985), Newbery (1970), Sheshinski (1972).
} 
be said in terms of standard welfare criteria if we use well-known practical indices as basic tools of distributional analysis.

\section{The Approach}

First, some notation and definitions.

Definition 1 The set of income distributions $X$ is the set of all ordered finitedimensioned non-negative vectors, excluding the zero vector.

In implementing this definition we adopt the convention that for any $\mathbf{x} \in X$ : $x_{1} \leq x_{2} \leq x_{3} \leq \ldots$ Also, for any $\mathbf{x} \in X$, denote the number of persons in the distribution as $n(\mathbf{x})$ and the arithmetic mean of the distribution as $\mu(\mathbf{x})$. Social welfare and inequality are given, respectively, by functions $W: X \rightarrow \Re$, $I: X \rightarrow \Re$. Economic meaning is given to $W$ and $I$ by a number of assumptions about their mathematical properties, many of which have come to be accepted as standard. Foremost among these are

Definition 2 The social welfare function is monotonic if, for any $\mathbf{x}, \mathbf{x}^{\prime} \in X$, $\mathbf{x}^{\prime}>\mathbf{x}$ implies $W\left(\mathbf{x}^{\prime}\right)>W(\mathbf{x}) .^{3}$

Definition 3 The social welfare function (resp. an inequality measure) satisfies the principle of transfers if, for any $\mathbf{x}, \mathbf{x}^{\prime} \in X$ and $\delta>0$ such that $x_{i}^{\prime}=x_{i}+\delta$, 3 " $\mathrm{x}^{\prime}>\mathrm{x}$ " means " $\mathrm{x}^{\prime} \geq \mathrm{x}, \mathrm{x}^{\prime} \neq \mathrm{x}$ ". 
$x_{j}^{\prime}=x_{j}-\delta, x_{k}^{\prime}=x_{k}, k \neq i, j$ and $x_{i}<x_{i}^{\prime} \leq x_{j}^{\prime}<x_{j} W\left(\mathbf{x}^{\prime}\right) \geq W(\mathbf{x})$ (resp. $\left.I\left(\mathbf{x}^{\prime}\right) \leq I(\mathbf{x})\right)$.

The property of monotonicity is of particular interest in view of its relationship to the Pareto principle - see Amiel and Cowell (1994b) - although it does not enjoy universal support as a welfare principle - see Amiel and Cowell (1994a), McClelland and Rohrbaugh (1978).

Definition 4 The class $\mathfrak{W}_{1}$ of social welfare functions consists of functions $W$ : $X \rightarrow \Re$ that satisfy the principles of monotonicity and transfers. If, for some $\mathbf{x}, \mathbf{x}^{\prime} \in X$, it is the case that $W(\mathbf{x})>W\left(\mathbf{x}^{\prime}\right)$ for all $W \in \mathfrak{W}_{1}$ we shall say that distribution $\mathbf{x}$ is $\mathfrak{W}_{1}$-preferable to $\mathbf{x}^{\prime}$.

We shall establish results for a fairly broad class of inequality indices. To introduce this we need the following two definitions:

Definition 5 For any $\mathbf{x} \in X$, the income share of person $i$ is:

$$
s_{i}(\mathbf{x}):=\frac{x_{i}}{n(\mathbf{x}) \mu(\mathbf{x})}
$$

Definition 6 An additive distributional-shares inequality index can be written as

$$
\sum_{i=1}^{n(\mathbf{x})} w_{i} \phi\left(s_{i}(\mathbf{x})\right)
$$




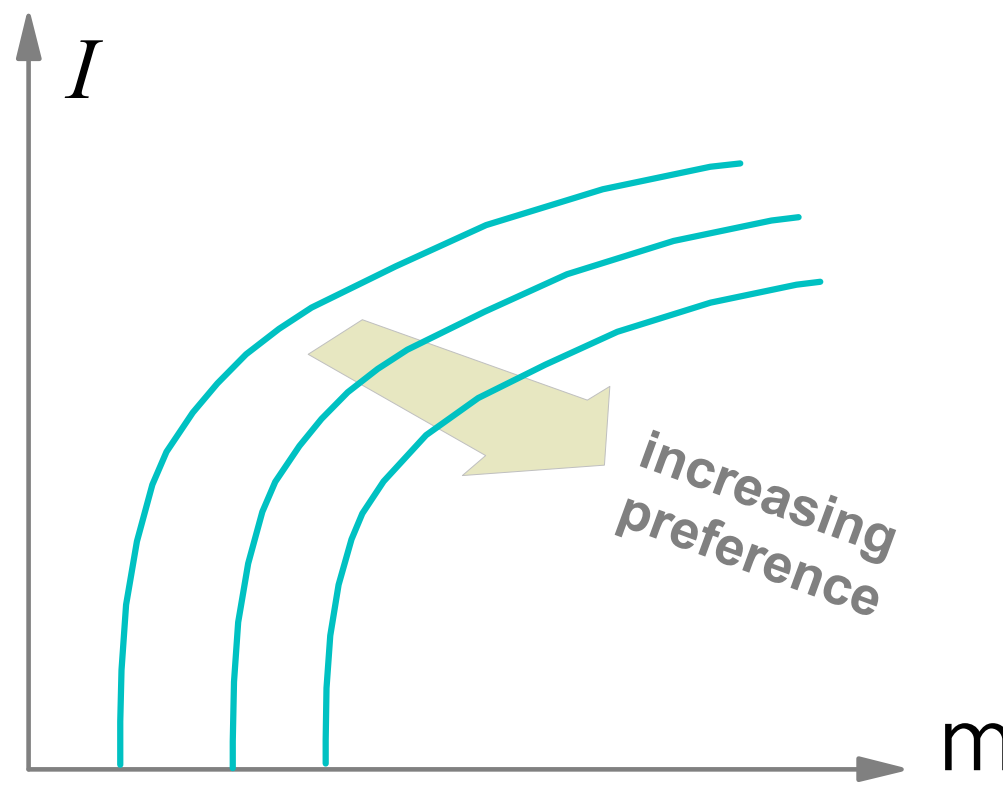

Figure 1: The Reduced-Form Social Welfare Function

where $w_{i}$ is a weight and $\phi:[0,1] \mapsto \Re$ is monotonic and convex. ${ }^{4}$

Examples of the ADSI class of indices include all the generalised-entropy indices, the Gini index, the relative mean deviation and the logarithmic variance. ${ }^{5}$ The results which follow will also apply to monotonic affine transforms of (2).

\footnotetext{
${ }^{4}$ The approach is based upon the so-called relative inequality aggregation property Blackorby and Donaldson (1984). Notice the essential difference from the standard welfare-theoretic approach to the definition of an inequality measure as in Atkinson (1970), Kolm (1969), Sen (1973) where the specification of the welfare function or of equally-distributed equivalent income usually forces monotonicity on the part of the social welfare function - see also Ebert (1988).

${ }^{5}$ This is distinct from the variance of logarithms - see Cowell (1995).
} 


\subsection{The Reduced-form SWF}

It is common to find the social welfare function written in a "reduced form" as a function of mean income and inequality. ${ }^{6}$ To formalise this approach let us introduce the concept of a distributional profile $z:=(\mu, I)$, a mean-income and inequality pair. In this interpretation the admissible set of profiles $Z \subseteq \Re_{+}^{2}$ may be written

$$
Z:=\{(\mu, I): \mu \geq 0,0 \leq I \leq \bar{I}\}
$$

where $\bar{I}$ is the least upper bound on $I$. Then the reduced-form SWF $H^{(W)}: Z \rightarrow$ $\Re$ is given by

$$
W(\mathbf{x})=H^{(W)}(\mu(\mathbf{x}), I(\mathbf{x}))
$$

where $H^{(W)}$ is non-decreasing in its first argument and non-increasing in its second argument.

Where there is no problem of ambiguity we will drop the superscript on $H$. Notice that the adoption of this approach implies that two distributions with same $z$-profile will be regarded as equivalent in welfare terms, and that the tradeoff implicit in the use of $H$ is only well-defined for a particular cardinalisation of the inequality index $I$. We shall assume that the issue of the appropriate cardinalisation has been settled by criteria which are not of concern to the present study.

\footnotetext{
${ }^{6}$ This is the term used by Champernowne and Cowell (1997). See also the term "abbreviated social-welfare function" used in Lambert (1993), Chapter 5.
} 


\section{Differentiable Social-welfare Functions}

The ADSI class of measures contains a number of well known indices and yields some readily interpretable results. To see this let us endow $I$ with the elementary cardinalisation of (2). Substituting from (2) into (4) we have

$$
W(\mathbf{x})=H\left(\mu(\mathbf{x}), \sum_{i=1}^{n(\mathbf{x})} w_{i} \phi\left(s_{i}(\mathbf{x})\right)\right)
$$

Assume further that $W$ is differentiable. Then differentiating (5) with respect to $x_{i}$ we have:

$$
n(\mathbf{x}) \frac{\partial W(\mathbf{x})}{\partial x_{i}}=H_{\mu}+H_{I}\left[\frac{w_{i} \phi_{s}\left(s_{i}(\mathbf{x})\right)}{\mu(\mathbf{x})}-\frac{\sum_{j=1}^{n(\mathbf{x})} w_{j} s_{j}(\mathbf{x}) \phi_{s}\left(s_{j}(\mathbf{x})\right)}{\mu(\mathbf{x})}\right]
$$

where the relevant partial derivatives are denoted by subscripts. From (6) the following result is immediate

Theorem 1 Given a differentiable reduced form social-welfare function $H$ the underlying individualistic welfare function satisfies monotonicity if and only if the following condition on the slope of the welfare-contour is satisfied:

$$
-\frac{H_{\mu}}{H_{I}}>\max \left\{\frac{w_{i} \phi_{s}\left(s_{i}(\mathbf{x})\right)-\sum_{j=1}^{n(\mathbf{x})} w_{j} s_{j}(\mathbf{x}) \phi_{s}\left(s_{j}(\mathbf{x})\right)}{\mu(\mathbf{x})}\right\}
$$




\subsection{Applications}

The condition (7) can be easily interpreted in the case of the Generalised Entropy index:

$$
-\frac{H_{\mu}}{H_{I}}>\max \frac{\frac{\left[n(\mathbf{x}) s_{i}(\mathbf{x})\right]^{\alpha-1}-1}{\alpha-1}-\alpha I(\mathbf{x})}{n(\mathbf{x}) \mu(\mathbf{x})}
$$

and the relative mean deviation:

$$
-\frac{H_{\mu}}{H_{I}}>\frac{\frac{2 m(\mathbf{x})+1}{n(\mathbf{x})}-I(\mathbf{x})}{n(\mathbf{x}) \mu(\mathbf{x})}
$$

where $m(\mathbf{x})=\#\left\{x_{i} \leq \mu(\mathbf{x})\right\}$. The interpretation of these results will be discussed below.

\subsection{The Gini Index}

The Gini index $G(\mathbf{x})$ can also be obtained as a special case of (7) by rewriting. it as: ${ }^{7}$

$$
G(\mathbf{x})=\sum_{i=1}^{n(\mathbf{x})} w_{i} s_{i}
$$

where

$$
w_{i}=\frac{2 i-n(\mathbf{x})-1}{n(\mathbf{x})}
$$

We will call the extended Gini subclass those inequality measures of the form (10) but with the restriction (11) replaced by the mild condition that $w_{i}$ be increasing

\footnotetext{
${ }^{7}$ See, for example Cowell (1995), Sen (1973).
} 
with $i{ }^{8}$ Notice that for the extended Gini and the true Gini $\phi_{s}=1$ wherever the differential is defined. Also note that for the true Gini $\max w_{i}=\frac{n(\mathbf{x})-1}{n(\mathbf{x})}$ which for large populations becomes 1 ; in this limiting case the right-hand side of (7) becomes:

$$
\frac{1-G(\mathbf{x})}{\mu(\mathbf{x})}
$$

However in the case of the Gini index the differentiability assumption used in Theorem 1 will be inappropriate. For this reason and for the reason that apart from mathematical tractability - differentiability has little to recommend it in social-welfare analysis, we seek a more general characterisation of the main point.

\section{A General Result}

To avoid the over-simplification associated with the differentiability assumption consider the general shape properties of the reduced-form social-welfare function at any arbitrary point in the set of all profiles $Z$.

For any distributional profile $z_{0} \in Z$ it is clear that any point in

$$
A\left(z_{0}\right):=\left\{z \in Z: \mu \geq \mu_{0}, I \leq I_{0}, z \neq z_{0}\right\}
$$

\footnotetext{
${ }^{8}$ See for example the contributions by Donaldson and Weymark (1980, 1983), Weymark (1981), , Yitzhaki (1983). However note that Chakravarty's extended Gini does not fit within this category (Chakravarty 1988).
} 
will be regarded as $\mathfrak{W}_{1}$-preferable to $z_{0}$. However, we can be more precise about the set of $\mathfrak{W}_{1}$-preferable profiles. First define the set of income distributions consistent with any profile $z_{0} \in Z$ :

$$
X\left(z_{0}\right):=\left\{\mathbf{x}: \mathbf{x} \in X, \mu(\mathbf{x})=\mu_{0}, I(\mathbf{x})=I_{0}\right\}
$$

and a conditional upper-bound value of inequality, of a given population size $n=n(\mathbf{x}):$

$$
I^{*}\left(\mu ; z_{0}\right):=\max _{\mathbf{x} \in X\left(z_{0}\right)} I\left(\mathbf{x}+\iota_{n} n\left[\mu-\mu_{0}\right]\right)
$$

where $\boldsymbol{\iota}_{n}:=(0,0, \ldots, 0,1)$. These constructs then permit the definition of the following subset of $A\left(z_{0}\right)$ :

$$
P\left(z_{0}\right):=\left\{z \in Z: \mu \geq \mu_{0}, I \leq I^{*}\left(\mu ; z_{0}\right), z \neq z_{0}\right\}
$$

Theorem $2 \forall W \in \mathfrak{W}_{1}, \forall z_{1} \in P\left(z_{0}\right)$ :

$$
H^{(W)}\left(z_{1}\right)>H^{(W)}\left(z_{0}\right)
$$

Proof. For some $z_{0} \in Z$ and some $z_{1}:=\left(\mu_{1}, I_{1}\right) \in P\left(z_{0}\right)$, given that $\mu_{0}<\mu_{1}$ by construction, if $I_{0} \geq I_{1}$ then it is trivial to show that (17) holds. So take the 


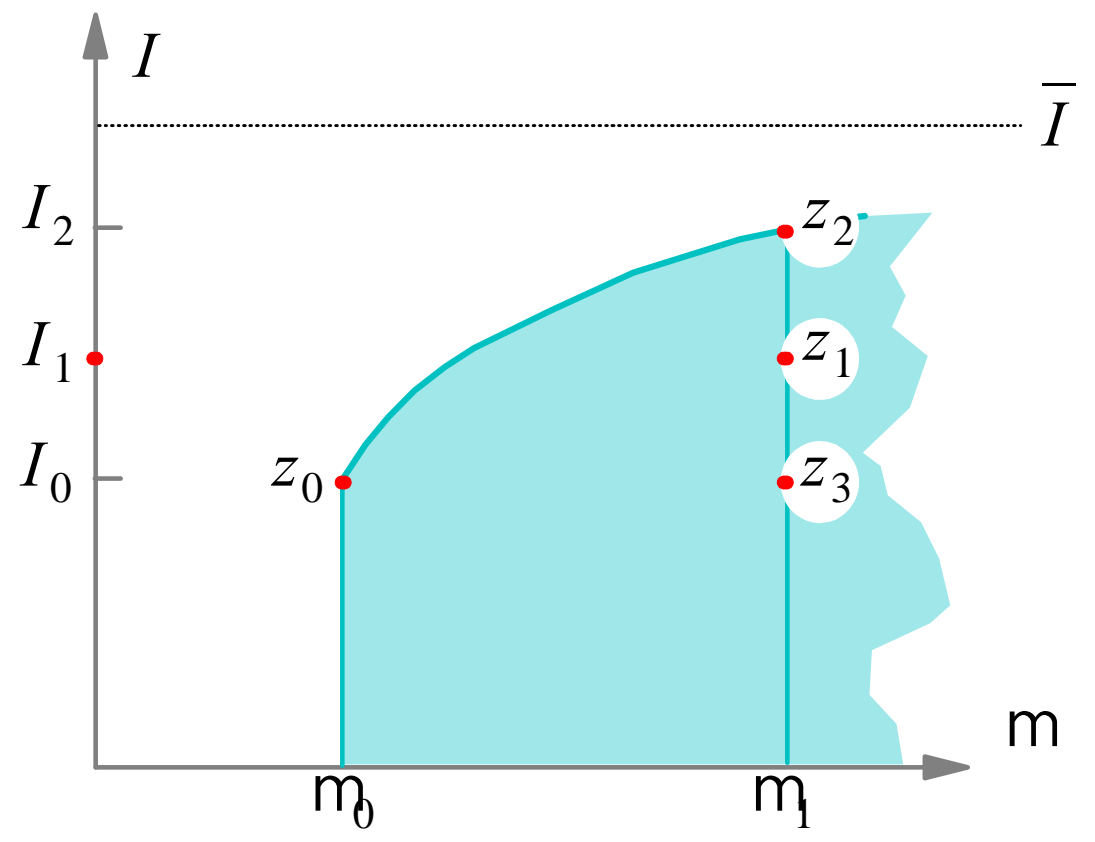

Figure 2: The set $P\left(z_{0}\right)$

more interesting subcase where $I_{0}<I_{1}$, and define

$$
\begin{gathered}
\mathbf{x}_{2}:=\arg \max _{\mathbf{x} \in X\left(z_{0}\right)} I\left(\mathbf{x}+\iota_{n} n\left[\mu_{1}-\mu_{0}\right]\right) \\
\mathbf{x}_{3}:=\frac{\mu_{1}}{\mu_{0}} \mathbf{x}_{0}
\end{gathered}
$$

The profiles $z_{2}$ and $z_{3}$ corresponding to $\mathbf{x}_{2}$ and $\mathbf{x}_{3}$ are illustrated in Figure 2 .

Noting that $W\left(\mathbf{x}_{2}\right)>W\left(\mathbf{x}_{0}\right)$ we have

$$
\begin{gathered}
\forall \alpha \in[0,1]: W\left(\alpha \mathbf{x}_{2}+[1-\alpha] \mathbf{x}_{3}\right)>W\left(\mathbf{x}_{0}\right), \\
\forall \alpha \in[0,1]: \mu\left(\alpha \mathbf{x}_{2}+[1-\alpha] \mathbf{x}_{3}\right)=\mu_{1}>\mu\left(\mathbf{x}_{0}\right)=\mu_{0},
\end{gathered}
$$


and by the principle of transfers $I^{*}\left(\mu ; z_{0}\right)=I\left(\mathbf{x}_{2}\right) \geq I_{1} \geq I\left(\mathbf{x}_{3}\right)=I_{0}$. By continuity there must be some $\alpha \in[0,1]$ such that $^{9}$

$$
I\left(\alpha \mathbf{x}_{2}+[1-\alpha] \mathbf{x}_{3}\right)=I_{1}
$$

in which case it is immediate from (20) that (17) holds.

Notice that, by construction, $P\left(z_{0}\right)$ is the largest set of profiles which are regarded as $\mathfrak{W}_{1}$-preferable to $z_{0}$, and that it is bounded by the graph of $I^{*}$ : the set of points of the form $\bar{P}\left(z_{0}\right):=\left\{z: z=\left(\mu, I^{*}\left(\mu ; z_{0}\right)\right), \mu \geq \mu_{0}\right\}$ provides a boundary conditional on a particular distributional profile $z_{0}$. For the ADSI class we have

$$
I^{*}\left(\mu ; z_{0}\right):=\sum_{i=1}^{n-1} w_{i}\left[\phi\left(\frac{x_{i}}{n \mu}\right)\right]+w_{n}\left[\phi\left(\frac{x_{n}+n\left[\mu-\mu_{0}\right]}{n \mu}\right)\right]
$$

which, in the case of the subclass of extended Gini measures, reduces to

$$
I^{*}\left(\mu ; z_{0}\right):=\bar{I}-\left[\bar{I}-I_{0}\right] \frac{\mu_{0}}{\mu}
$$

Notice that through each point (profile) $z_{0} \in Z$, there is a frontier $\bar{P}$ and a contour of the reduced-form social welfare function $H^{(W)}$. We may then state:

Theorem 3 For any inequality-averse monotonic $S W F$ and any $z_{0} \in Z$, when-

\footnotetext{
${ }^{9}$ See the profile $z_{1}$ in Figure 2. Notice that $I$ must be continuous in view of the convexity of $\phi$.
} 


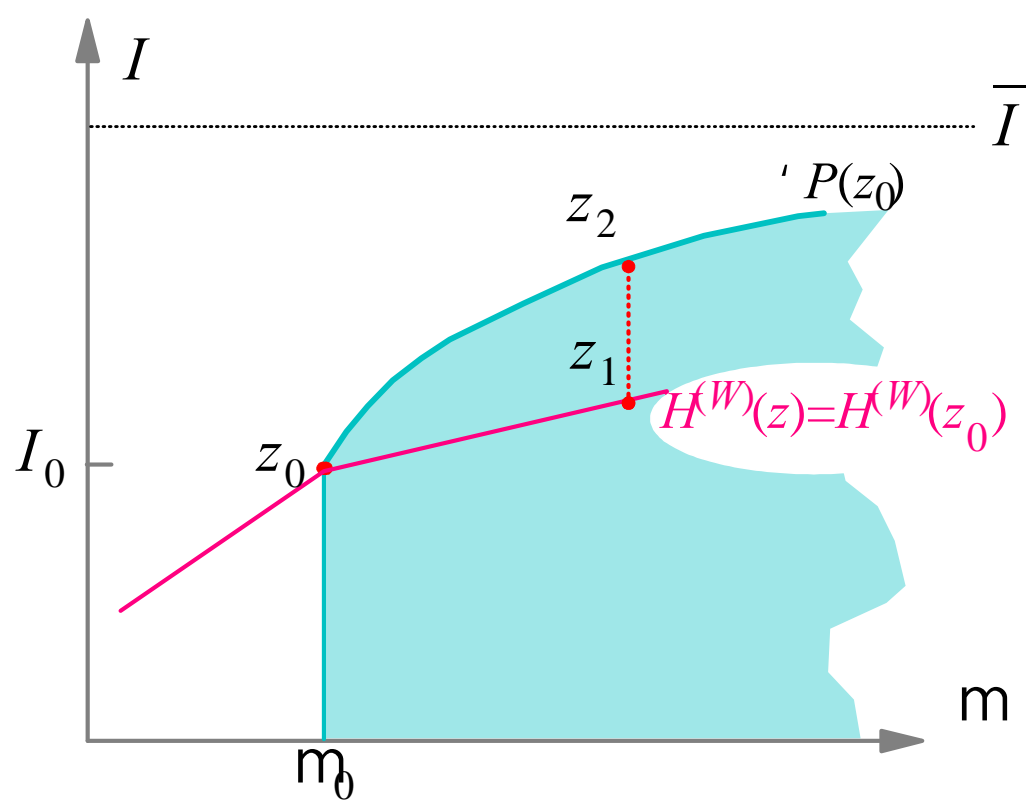

Figure 3: A violation of monotonicity

ever the contour of $H^{(W)}$ intersects $\bar{P}\left(z_{0}\right)$, it must do so from below.

Proof. Suppose to the contrary that $H^{(W)}$ intersects $\bar{P}\left(z_{0}\right)$ from above as in Figure 3. ${ }^{10}$ By construction $H^{(W)}\left(z_{0}\right)=H^{(W)}\left(z_{1}\right), z_{2}$ is $\mathfrak{W}_{1}$-preferable to $z_{0}$. and profiles $z_{1}, z_{2}$ have the same mean. If $W$ is inequality-averse then $H^{(W)}\left(z_{2}\right)>H^{(W)}\left(z_{1}\right)=H^{(W)}\left(z_{0}\right)$ which means that $W$ must violate monotonicity - a contradiction.

This theorem may then be used to give a more general interpretation to Theorem 1 which was obtained under the assumption of uniform differentiability:

\footnotetext{
${ }^{10}$ The $H^{(W)}$-contour has been drawn with a kink at $z_{0}$ to illustrate the generality of the result. Of course it need not be kinked, nor piecewise linear, and it may be convex rather than concave.
} 
Corollary 4 If $W$ satisfies monotonicity, the limit of the slope of $I^{*}\left(\mu ; z_{0}\right)$ as $\mu \downarrow$ $\mu_{0}$ must be less than the limit of the slope of the contour of $H^{(W)}(z)=H^{(W)}\left(z_{0}\right)$ as $\mu \downarrow \mu_{0}$.

Substitution from (23) or from (24) will confirm the result for the general ADSI class and for the special case of the Gini index respectively. In the latter case we find that the condition for monotonicity amounts to:

$$
-\frac{H_{\mu}^{(W)}}{H_{I}^{(W)}}>\frac{1-I}{\mu}
$$

where the slope is to be interpreted in the manner of Corollary 4. This can be reexpressed as a requirement on the reduced-form SWF in terms of the associated equality index $1-I$ : it should have the property that the elasticity of equality with respect to mean income be less than unity. The condition (25) can then be used to check the properties of reduced-form Gini-SWFs presented in the literature. Examples of measures satisfying the elasticity condition include $n^{2}[1-$ $I] \mu\left(\right.$ Sheshinski 1972) ${ }^{11}, \log \mu-I\left(\right.$ Katz 1972) and $\frac{\mu}{1+I}($ Kakwani 1986). However $\frac{1-I}{1+I} \mu$ (Chipman 1974, Dagum 1990) does not satisfy the condition and it is easy to see that this index violates monotonicity: Table 1 shows an example where distribution $\mathrm{B}$ has a higher mean income and higher inequality than $\mathrm{A}$. B also first-order dominates A. However the Chipman-Dagum social welfare function

\footnotetext{
${ }^{11}$ A generalisation of this form is to be found in Lambert (1985). See also Ben Porath and Gilboa (1994)
} 


\begin{tabular}{rll} 
& $\mathbf{A}$ & $\mathbf{B}$ \\
\hline$x_{1}$ & 4500 & 4600 \\
$x_{2}$ & 4000 & 4000 \\
$x_{3}$ & 3500 & 3500 \\
& & \\
\hline$\mu(\mathbf{x})$ & 4000 & 4033 \\
$I(\mathbf{x})$ & 0.056 & 0.061 \\
$W(\mathbf{x})=\frac{1-I}{1+I} \mu$ & 3579 & 3572 \\
\hline
\end{tabular}

Table 1: The Gini coefficient $I$ and the Chipman-Dagum Welfare Index $W$ indicates that society is worse-off in B than in A.

Similar interpretations can be provided in terms of other members of the ADSI class (2).

\section{Conclusions}

The use of ad hoc inequality measures begs the question of whether the empirical judgements made using them will be in accordance with conventional welfare properties. The reduced-form SWF is the appropriate tool to clarify this issue. The transfer principle is inherited automatically by the SWF from an inequality measure; now we have shown that the condition on the reduced-form SWF that ensures consistency with the monotonicity criterion is surprisingly simple. In the case of the Gini index this condition is particularly appealing, and does not rely on arbitrary assumptions of differentiability of the SWF.

Finally, a word of caution. Cardinalisations of inequality measures are sometimes viewed as irrelevant, but in the analysis of the so-called "equity-efficiency 
trade-off" this is not quite so. As the discussion of the elasticity of the $H^{(W)}$ -contours makes clear, the results we have obtained are specifically for the "natural" cardinalisation of the index (2). A modified version of Theorem 1 and related results will be applicable under continuous monotonic transformations of $I$. 


\section{References}

Amiel, Y. and F. A. Cowell (1994a). Income inequality and social welfare. In J. Creedy (Ed.), Taxation, Poverty and Income Distribution, pp. 193-219. Edward Elgar.

Amiel, Y. and F. A. Cowell (1994b). Monotonicity, dominance and the Pareto principle. Economics Letters 45, 447-450.

Atkinson, A. B. (1970). On the measurement of inequality. Journal of Economic Theory 2, 244-263.

Ben Porath, E. and I. Gilboa (1994). Linear measures, the Gini index, and the income-equality trade-off. Journal of Economic Theory 64, 443-467.

Blackorby, C. and D. Donaldson (1978). Measures of relative equality and their meaning in terms of social welfare. Journal of Economic Theory 18, 59-80.

Blackorby, C. and D. Donaldson (1980). A theoretical treatment of indices of absolute inequality. International Economic Review 21, 107-136.

Blackorby, C. and D. Donaldson (1984). Ethically significant ordinal indices of relative inequality. In R. L. Basmann and G. G. Rhodes (Eds.), Advances in Econometrics, Volume 3, pp. 83-6. JAI Press.

Chakravarty, S. R. (1988). Extended Gini indices of inequality. International Economic Review 29(2), 147-156.

Champernowne, D. G. and F. A. Cowell (1997). Inequality and Income Distri- 
bution. Cambridge: Cambridge University Press.

Chipman, J. S. (1974). The welfare ranking of Pareto distributions. Journal of Economic Theory 9, 275-282.

Cowell, F. A. (1995). Measuring Inequality (Second ed.). Hemel Hempstead: Harvester Wheatsheaf.

Dagum, C. (1990). On the relationship between income inequality measures and social welfare functions. Journal of Econometrics 43, 91-102.

Donaldson, D. and J. A. Weymark (1980). A single parameter generalization of the Gini indices of inequality. Journal of Economic Theory 22, 67-68.

Donaldson, D. and J. A. Weymark (1983). Ethically flexible Gini indices for income distribution in the continuum., Journal of Economic Theory 29(4), $353-358$.

Ebert, U. (1988). Measurement of inequality: an attempt at unification and generalization. Social Choice and Welfare 5, 147-169.

Kakwani, N. C. (1986). Analyzing Redistribution Policies. Cambridge University Press.

Katz, A. (1972). On the social welfare function and the parameters of income distribution. Journal of Economic Theory 5, 377-382.

Kolm, S. C. (1969). The optimal production of social justice. In J. Margolis and H. Guitton (Eds.), Public Economics. London: Macmillan. 
Kolm, S. C. (1976a). Unequal inequalities I. Journal of Economic Theory 12, 416-442.

Kolm, S. C. (1976b). Unequal inequalities II. Journal of Economic Theory 13, $82-111$.

Kondor, Y. (1975). Value judgement implied by the use of various measures of income inequality. The Review of Income and Wealth, 309-321.

Lambert, P. J. (1985). Social welfare and the Gini coefficient revisited. Mathematical Social Science 9, 19-26.

Lambert, P. J. (1993). The Distribution and Redistribution of Income (Second ed.). Manchester, UK: Manchester University Press.

McClelland, G. and J. Rohrbaugh (1978). Who accepts the Pareto axiom? the role of utility and equity in arbitration decisions. Behavioural Science 23, $446-456$.

Newbery, D. M. G. (1970). A theorem on the measurement of inequality. Journal of Economic Theory 2, 264-266.

Sen, A. K. (1973). On Economic Inequality. Oxford: Clarendon Press.

Sheshinski, E. (1972). Relation between a social welfare function and the Gini index of inequality. Journal of Economic Theory 4, 98-100.

Weymark, J. A. (1981). Generalized Gini inequality indices. Mathematical Social Sciences 1, 409-430. 
Yitzhaki, S. (1983). On an extension of the Gini inequality index. International Economic Review 24(10), 617-628. 